Military Technical College Kobry El-Kobbah, Cairo, Egypt

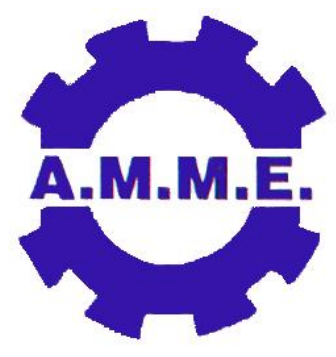

$14^{\text {th }}$ International Conference on Applied Mechanics and Mechanical Engineering.

\title{
Frequency optimization of functionally graded bars in axial motion
}

\author{
By \\ Karam Y. Maalawi*
}

\begin{abstract}
:
This paper presents an exact method for maximizing the natural frequencies of functionally graded material (FGM) bars in axial motion. To satisfy the economic feasibility requirements, the total structural mass is maintained at a constant value equal to that of a defined baseline design. The composition of the material of construction is optimized by defining the spatial distribution of volume fractions of the material constituents using either continuous or discrete distributions along the bar length. The major aim is to tailor the material distribution in the axial direction so as to maximize the frequencies and place them at their target values to avoid the occurrence of large amplitudes of vibration without the penalty of increasing structural mass. The resulting optimization problem has been formulated as a nonlinear mathematical programming problem solved by invoking the MatLab optimization toolbox routines, which implement the method of feasible directions interacting with the associated eigenvalue problem routines. As a case study, a bar with Fixed-Fixed boundary condition has been thoroughly investigated. It was shown that the use of material grading concept can be promising in maximizing the natural frequencies and producing efficient economical designs having optimal stiffness and mass distributions as compared with their corresponding baseline designs.
\end{abstract}

\section{Keywords:}


Functionally graded materials, axial vibration, composite structures and optimum design

\section{* Mechanical Engineering, National Research Center, Dokki, Cairo, Egypt. \\ 1. Introduction:}

The concept of functionally graded materials (FGMs), in which the properties vary spatially within a structure, was originated in Japan in 1984 during the space project, in the form of proposed thermal barrier material capable of withstanding high temperature gradients. FGMs may be defined as advanced composite materials that fabricated to have graded variation of the relative volume fractions of the constituent materials [1]. FGMs can be promising in several applications such as, spacecraft heat shields, high performance structural elements and critical engine components. A few studies have addressed the dynamics of FGM structures. Loy et al. [2] considered vibration analysis of $F G M$ cylindrical shells composed of stainless steel and nickel, which were graded in thickness direction according to a volume fraction power-law distribution. Closed-form solutions for calculating the natural frequencies of an axially graded beam were derived by Elishakoff and Guede [3], where the modulus of elasticity was taken as a polynomial of the axial coordinate along the beam's length. Another work by Vel and Batra [4] presented a three-dimensional exact solution for free and forced vibrations of simply supported FGM rectangular plates. Considering, next, structural dynamic optimization, Masad [5] presented an efficient numerical approach for computing optimum shapes of one-dimensional structural models for maximum fundamental frequency. Another work by Maalawi [6] addressed the problem of frequency maximization of rods in axial motion under mass constraint. The dual problem of mass minimization under frequency constraint was also addressed, and results were presented and discussed for both continuous and discrete multi-element structural models. In the field of the optimum design of FGMstructures, Qian and Batra [7] considered frequency optimization of a cantilevered plate with variable volume fraction according to simple power-laws. They implemented genetic algorithms to find the optimum values of the power exponents, which maximize the natural frequencies. Goupee and Vel [8] proposed a methodology to optimize the natural frequencies of functionally graded beam with variable volume fraction of the constituent materials in the beam's length and height directions. They used a piecewise bi-cubic interpolation of volume fraction values specified at a finite number of grid points. Librescu and Maalawi [9] introduced the underlying concepts of using material grading in optimizing subsonic wings against torsional instability, where both continuous and piecewise structural models were successfully implemented. The aim of the present study is to incorporate the effect of changing the volume fractions of the constituent materials for maximizing the natural frequencies of an axially-vibrating bar with a constant total mass. The proposed optimization 
model can be regarded as a useful tool in obtaining bar designs having enhanced dynamic performance.

\section{The eigenvalue problem:}

The associated eigenvalue problem of the free axial motion of a vibrating bar is described by the differential equation [10]:

$$
\frac{d}{d x}\left[E A(x) \frac{d U}{d x}\right]+\rho A(x) \omega^{2} U(x)=0
$$

which must be satisfied over the entire bar's length (L). In equation (1), $x$ denotes the axial coordinate, $U(x)$ spatial dependence of the axial displacement at any position $x, E$ modulus of elasticity, $A$ cross-sectional area, $\omega$ natural circular frequency and $\rho$ mass density. The associated boundary conditions for the case of Fixed-Fixed bars are:

$$
U(O)=0 \quad \text { and } \quad U(L)=0
$$

Next, it is essential to appropriately define a baseline design to which the resulting optimal solutions can be compared. The baseline design has been selected to be a composite bar made of two different materials denoted by $(A)$ and $(B)$ and has uniform mass and stiffness distributions along its length, with equal volume fractions $(V)$ of the constituent materials, i.e. $V_{A}=V_{B}=50 \%$. It is assumed that the optimized designs shall have the same total mass, length, cross sectional area and shape, type of material of construction and type of boundary conditions of those of the known baseline design. For the optimized designs, the physical and mechanical properties are allowed to vary lengthwise, yielding to grading of the material in the direction of the bar's axis. Assuming no voids are present, the distributions of the mass density $(\rho)$ and modulus of elasticity $(E)$ are determined as follows [11]:

Volume fractions $\quad: V_{A}(x)+V_{B}(x)=1$

Mass density $\quad: \rho(x)=V_{A}(x) \rho_{A}+V_{B}(x) \rho_{B}$

Modulus of elasticity: $E(x)=V_{A}(x) E_{A}+V_{B}(x) E_{B}$

Normalizing with respect to the baseline design by dividing equation (1) by $\left(E_{o} A / L\right)$ and differentiating once more with respect to $x$, we get:

$$
\hat{E} \frac{d^{2} \hat{U}}{d \hat{x}^{2}}+\frac{d \hat{E}}{d \hat{x}} \cdot \frac{d \hat{U}}{d \hat{x}}+\hat{\rho} \hat{\omega}^{2} \hat{U}=0
$$

which must be satisfied on the interval $0<\hat{x}<1$. The various dimensionless quantities denoted by $\left({ }^{\wedge}\right)$ are defined in Table 1 . Since the total structural mass $M_{s}$ is kept equal to that of the baseline design (i.e. $\hat{M}_{s}=1$ ), therefore, a feasible design should satisfy the following dimensionless mass equality constraint:

$\int_{0}^{1} V_{A}(\hat{x}) d \hat{x}=0.5$

Table (1): Definition of dimensionless quantities

\begin{tabular}{|l|l|}
\hline Quantity & Dimensionless Notation \\
\hline Axial coordinate & $\hat{x}=x / L$ \\
\hline
\end{tabular}




\begin{tabular}{|l|l|}
\hline Longitudinal displacement & $\hat{U}=U / L$ \\
\hline Modulus of elasticity & $\hat{E}=E / E_{o}$ \\
\hline Mass density & $\hat{\rho}=\rho / \rho_{o}$ \\
\hline Total structural mass & $\hat{M}_{S}=\int_{0}^{l} \hat{\rho} d \hat{x}$ \\
\hline Circular frequency & $\hat{\omega}=\omega L \sqrt{\rho_{o} / E_{o}}$ \\
\hline
\end{tabular}

* The baseline design parameters are: $L=$ total length, $\mathrm{A}=$ cross-sectional area, $E_{o}=0.5\left(E_{A}+E_{B}\right), \rho_{o}=0.5\left(\rho_{A}+\rho_{B}\right)$.

\section{Solution procedures:}

\subsection{Continuous model:}

The general solution of Eq. (4) can be expressed by the following power series [12]

$$
\hat{U}(\hat{x})=\sum_{m=1}^{2} C_{m} \lambda_{m}(\hat{x})
$$

where $C_{m}$ 's are the constants of integration and $\lambda_{m}$ 's are two linearly independent solutions that have the form:

$$
\lambda_{m}(\hat{x})=\sum_{n=m}^{\infty} a_{m n} \hat{x}^{n-1} \quad(n \geq m)
$$

The unknown coefficients $a_{m n}$ can be determined by substitution into the differential equation (4) and equating coefficients of like powers of $\hat{x}$. Variation of the volume fractions in FGM structures is usually described by power-law distributions [3]. In the present study, linear and parabolic model types are considered with their corresponding derived recurrence formulas given in the following:

Linear distribution (Symmetrical about $\hat{x}=1 / 2): \quad V_{A}(\hat{x})=V_{A}(0)-2 \Delta V \hat{x}, \Delta V=V_{A}(0)-V_{A}(1 / 2)$ $a_{m, n}=\frac{2 \gamma(n-2)^{2} a_{m, n-1}-\theta \hat{\omega}^{2} a_{m, n-2}+2 \xi \hat{\omega}^{2} a_{m, n-3}}{\beta(n-1)(n-2)}$

Parabolic distribution (Symmetrical about $\hat{x}=1 / 2): V_{A}(\hat{x})=V_{A}(0)+4 \Delta V\left(\hat{x}^{2}-\hat{x}\right), \Delta V=V_{A}(0)$ $V_{A}(1 / 2)$

$a_{m, n}=\frac{4 \gamma(n-2)^{2} a_{m, n-1}-\left[4 \gamma(n-2)(n-3)+\theta \hat{\omega}^{2}\right] a_{m, n-2}+4 \xi \hat{\omega}^{2}\left(a_{m, n-3}-a_{m, n-4}\right)}{\beta(n-1)(n-2)}$

The Greek symbols in equations (8) and (9) are defined in the following:

$$
\begin{aligned}
& \beta=\hat{E}_{B}+\Delta \hat{E} V_{A}(0) \\
& \gamma=\Delta \hat{E} \Delta V, \quad \Delta \hat{E}=\left(\hat{E}_{A}-\hat{E}_{B}\right)
\end{aligned}
$$




$$
\begin{aligned}
& \theta=\hat{\rho}_{B}+\Delta \hat{\rho} \quad V_{A}(0) \\
& \xi=\Delta \hat{\rho} \Delta V, \quad \Delta \hat{\rho}=\left(\hat{\rho}_{A}-\hat{\rho}_{B}\right)
\end{aligned}
$$

A coefficient $a_{m, n}$ is set equal to zero whenever $n$ is less than $m$, and the leading coefficients $a_{m, m}$ in each series are arbitrary and can be set equal to one. Table 2 summarizes the appropriate mathematical expressions of the associated frequency equations.

Table (2): Frequency equations for symmetrical material distribution

\begin{tabular}{|l|l|c|}
\hline Mode of vibration & Frequency equation & $\hat{\omega}_{0}$ \\
\hline Symmetrical modes: $\hat{U}(0)=\hat{U}^{\prime}(1 / 2)=0$ & $\sum_{n=3}^{\infty} a_{2 n}(n-1) /(2)^{n-2}=-1$ & $\pi$ \\
\hline Unsymmetrical modes: $\hat{U}(0)=\hat{U}(1 / 2)=0$ & $\sum_{n=2}^{\infty} a_{2 n} /(2)^{n-1}=0$ & $2 \pi$ \\
\hline
\end{tabular}

$\hat{\omega}_{\mathrm{o}}$ is the dimensionless fundamental frequency of the baseline design.

The notation ( )' means $d / d \hat{x}$.

\subsection{Piecewise model:}

A piecewise model concept was introduced by Maalawi [6] to obtain global optimal frequency designs of isotropic bars with piecewise uniform sections. Figure 1 shows an elastic, slender bar with total dimensionless length of unity constructed from any arbitrary number of uniform segments $\left(N_{s}\right)$, each of which has the same cross-sectional area but different properties of the material of construction. Such a configuration results in a piecewise axial grading of the material in the direction of the bar axis.

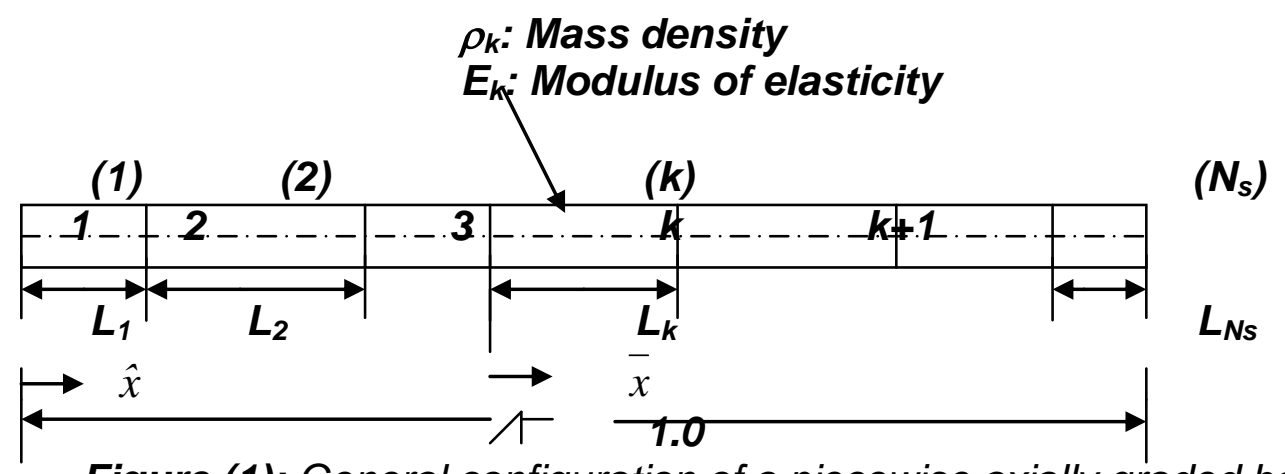

Figure (1): General configuration of a piecewise axially graded bar

For the $K$-th segment, Equation (4) reduces to

$$
\frac{d^{2} \hat{U}}{d \bar{x}^{2}}+\alpha_{k}^{2} \hat{U}=0, \alpha_{k}=\hat{\omega} \sqrt{\hat{\rho}_{k} / \hat{E}_{k}}
$$

where $\hat{E}_{k}\left(=E_{k} / E_{0}\right)$ is the dimensionless modulus of elasticity of the $k$-th segment. Equation (12) must be satisfied in the interval $0 \leq \overline{\mathrm{x}} \leq \hat{L}_{k}$, where $\overline{\mathrm{x}}=\hat{x}-\hat{x}_{k}$ and $\hat{L}_{k}=\left(L_{k} / L\right)$. Its general solution is: 


$$
\hat{U}(\bar{x})=C_{1} \operatorname{Sin}\left(\alpha_{k} \bar{x}\right)+C_{2} \operatorname{Cos}\left(\alpha_{k} \bar{x}\right)
$$

Expressing the constants of integration $C_{1}$ and $C_{2}$ in terms of the state variables $\left[\hat{U}, \hat{F}=\hat{E} \frac{d \hat{U}}{d \bar{x}}\right]$ at both ends of the $k$-th segment, we get:

$$
\left\{\begin{array}{c}
\hat{U}_{k+1} \\
\hat{F}_{k+1}
\end{array}\right\}=\left[T^{(k)}\right]\left\{\begin{array}{l}
\hat{U}_{k} \\
\hat{F}_{k}
\end{array}\right\}, \quad\left[T^{(k)}\right]=\left[\begin{array}{ll}
\operatorname{Cos} \alpha_{k} \hat{L}_{k} & \frac{\operatorname{Sin} \alpha_{k} \hat{L}_{k}}{\alpha_{k} \hat{E}_{k}} \\
-\alpha_{k} \hat{E}_{k} \operatorname{Sin} \alpha_{k} \hat{L}_{k} & \operatorname{Cos} \alpha_{k} \hat{L}_{k}
\end{array}\right]
$$

where $\left[T^{(k)}\right]$ is called the transfer matrix of the $k$-th segment. It is now possible to compute the state variables progressively along the bar length by applying continuity requirements among the interconnecting boundaries of the various bar segments. Therefore, the state variables at both ends of the bar can be related to each other through an overall transfer matrix denoted by $[T]$ :

$$
[T]=\left[T^{(N s)}\right]\left[T^{(N s-1)}\right] \ldots \ldots . .\left[T^{(2)}\right]\left[T^{(1)}\right]
$$

The required frequency equation for determining the natural frequencies can then be obtained by applying the associated boundary conditions (refer to Table 2) and considering only the nontrivial solution of the resulting matrix equation.

\section{Frequency optimization:}

Attractive goals of designing efficient structural members include minimization of structural weight, maximization of the natural frequencies and maximization of the critical buckling loads [13]. Direct maximization of the natural frequencies can ensure a simultaneous balanced improvement in both stiffness and structural mass distributions. The associated optimization problems are usually cast in nonlinear mathematical programming form [14] where the objective is to minimize a function $F(\underline{X})$ of a vector $\underline{X}$ of design variables, subject to certain number of constraints $G_{j}(\underline{X}) \leq 0, j=1,2, \ldots m$. In the present optimization problem, the objective function is represented by a direct maximization of a weighted-sum of the natural frequencies, which is expressed mathematically as follows:

Minimize $F(\underline{X})=-\sum_{i} \alpha_{i} \hat{\omega}_{i}$

$\sum_{i} \alpha_{i}=1, \quad 0 \leq \alpha_{i} \leq 1$

Subject to $\hat{M}_{s}=1$

where $\hat{\omega}_{i}$ are the normalized frequencies, $\alpha_{i}$ weighting factors measuring the relative importance of each frequency. For continuous models with known power-law distribution, $\underline{X}$ represents the volume fractions at the ends of the bar, while for discrete model $\underline{X}=\left(V_{A, k}, \hat{L}_{k}\right)_{k, 1,2, \ldots N s}$. Side constraints are always imposed on the design variables in order 
not to obtain unrealistic odd-shaped designs in the final optimum solutions. The MATLAB optimization toolbox is a powerful tool that includes many routines for different types of optimization encompassing both unconstrained and constrained minimization algorithms [14]. One of its useful routines is named "fmincon" which implements the method of feasible directions in finding the constrained minimum of an objective function of several variables.

\section{Results and discussions:}

The mathematical model developed above has been applied to obtain the required optimal solutions of FGM bars made of carbon-AS4 (material $A$ ) and epoxy-3501-6 (material $B$ ), which has favorable characteristics and is highly desirable in several mechanical, civil and aerospace engineering applications [11], refer to Table 3.

Table (3): Material properties of carbon-AS4 / epoxy-3501-6 composite

\begin{tabular}{|l|l|l|}
\hline Property & $\begin{array}{l}\text { Carbon Fibers } \\
\text { (material A) }\end{array}$ & $\begin{array}{c}\text { Epoxy Matrix } \\
\text { (material B) }\end{array}$ \\
\hline Mass density $\left(\mathrm{g} / \mathrm{cm}^{3}\right)$ & $\rho_{f}=1.81$ & $\rho_{m}=1.27$ \\
\hline Young's modulus $(\mathrm{GPa})$ & $E_{1 f}=235$ & $E_{m}=4.3$ \\
\hline Shear modulus $(\mathrm{GPa})$ & $G_{12 f}=27$ & $G_{m}=1.6$ \\
\hline Poisson's ratio & $v_{12 f}=0.2$ & $v_{m}=0.35$ \\
\hline
\end{tabular}

Considering first the piecewise model of a bar constructed from four segments with symmetry about the mid span, half of the bar can be analyzed with only four variables denoted by $\left(V_{A}, \hat{L}\right)_{k=1,2}$. Two variables can be eliminated using the equality constraints imposed on the total length and structural mass. Fig. 2 depicts the functional behavior of the dimensionless $1^{\text {st }}$ and $2^{\text {nd }}$ frequencies augmented with the equality mass constraint. It is seen that the functions are well behaved and continuous everywhere in the design space $\left(V_{A}-\hat{L}\right)_{1}$, except in the empty regions located at the upper right of the whole domain, where the mass equality constraint is violated. Such empty regions are bounded from below by the curve $\left(V_{A} \hat{L}\right)_{1}=0.5$; that is the volume fraction of material $(A)$ is equal to zero in the second segment (i.e. $V_{A, 2}=0$ ). The feasible domain in Fig. (2a) is seen to be split by the baseline contours $\left(\hat{\omega}_{1}=\pi\right)$ into two distinct zones. The one to the right encompasses the constrained global maxima, which is calculated to be $\hat{\omega}_{1, \max }=3.45406$ at the optimal design point $\left(V_{A}, \hat{L}\right)_{k=1,2}=(0.650,0.3375),(0.1885,0.1625)$. Actually, each design point inside the feasible domain corresponds to different material properties as well as different stiffness and mass distributions, while maintaining the total structural mass constant. Fig. (2b) shows the developed isomerits of the second frequency associated with the first unsymmetrical mode. Two global maxima can be observed having the calculated values $\hat{\omega}_{2, \max }=6.4732$ and 6.4711 , which correspond, respectively, to the design points $\left(V_{A}, \hat{L}\right)_{k=1,2}=(0.405,0.365),(0.757,0.135)$ located at the upper left region, and $(0.750$, $0.145),(0.3975,0.355)$ at the lower right region of the feasible domain. Table 4 summarizes the attained optimal solutions with increasing the number of segments. It is seen that the attained optimization gain (i.e. the percentage increase in the fundamental frequency above its baseline 
value) increases with the number of segments. However, it should be kept in mind that the cost of manufacturing will also be increased. Therefore, a compromise has to be made between the reduction of vibration and the cost of manufacturing. Another remarkable observation is that the attained optimal designs based on maximization of a single frequency do not guarantee maximization of other frequencies. For example, the design based upon maximization of $\hat{\omega}_{2}$ alone can result in a degraded value of the fundamental frequency $\left(\hat{\omega}_{1}=2.820\right)$, located in the region to the left of the design space, which is lower than that of the baseline design by about $10.24 \%$. Therefore, if one really seeks to maximize the overall stiffness-to-mass ratio of the vibrating rod, a multi-objective design optimization ought to be implemented instead. In this regard, the proper determination of the values of the weighting factors $\alpha_{i}$ ought to be based on the fact that each frequency shall be maximized from its initial value corresponding to the uniform baseline design. The appropriate values of the weighting factors were calculated to be: $\alpha_{i}=65.2 \%, 21.7 \% \quad 13.1 \%$. The attained optimal solutions for different optimization strategies are given in Table 5 for bars constructed from two segments. Considering next, the continuous distributions, Table 6 gives the attained optimal solutions for cases of linear and parabolic material grading. In both cases the total structural mass was maintained constant. As a general observation, patterns with higher fiber volume fraction near the fixed ends are always favorable. Maximization of the fundamental frequency alone produces an optimization gain of about $14.33 \%$ for the linear model with $0 \%$ and $100 \%$ volume fractions at the ends of the optimized bars. However, a drastic reduction in the $2^{\text {nd }}$ and $3^{\text {rd }}$ frequencies can be observed. Better solutions have been achieved by maximizing a weighted-sum of the first three frequencies, where the parabolic model was found to excel the linear one in producing balanced improvements in all frequencies. Results have also indicated that the Fixed-Fixed bars are recommended to have concave distribution rather than convex one. The latter produce poor patterns with degraded stiffness-to-mass ratio levels.
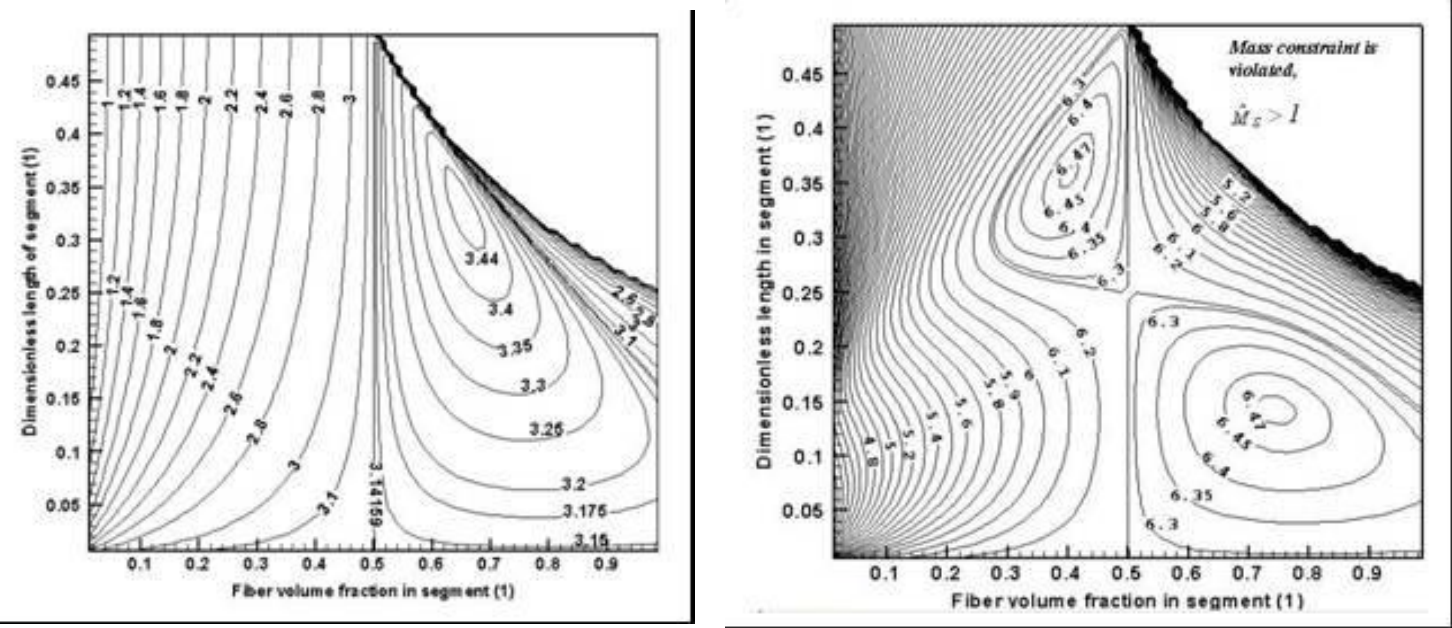

(a) Fundamental frequency (Symmetrical mode). (b) Second frequency (Unsymmetrical mode).

Figure (2): Level curves of the dimensionless frequency of a 4-segment fixed-fixed bar. Total structural mass is preserved: $\hat{M}_{s}=1.0$. 
Table (4): Optimal symmetrical patterns of FGM bars with Max. $\left(\hat{\omega}_{1}\right)$ under equality mass constraint.

\begin{tabular}{|l|l|l|l|}
\hline Ns & $\left(V_{A}, \hat{L}\right)_{k=1,2, . . N s}$ & $\hat{\omega}_{i}, i=1,2,3$ & $\%$ gain \\
\hline 1 & $(0.50,0.50)_{1}$ & $\begin{array}{l}(3.142,6.283, \\
9.425)\end{array}$ & $0 \%$ \\
& & $(3.454,5.537$, & $9.93 \%$ \\
\hline 2 & $(0.650,0.338)_{1},(0.189,0.163)_{2}$ & $8.081)$ & \\
\hline 3 & $(0.719,0.244)_{1},(0.413,0.144)_{2},(0.138,0.112)_{3}$ & $(3.531,5.487$, & $12.38 \%$ \\
& & $8.287)$ & \\
\hline 4 & $(0.750,0.23125)_{1},(0.425,0.13125)_{2},(0.225$, & $(3.562,5.133$, & $13.37 \%$ \\
& $0.07500)_{3},(0.0625,0.0625)_{4}$ & $8.231)$ & \\
\hline
\end{tabular}

Table (5): Optimal patterns of a two-segment FGM bar for different optimization strategies. Material distribution is symmetrical about the middle of bar length.

\begin{tabular}{|l|l|l|}
\hline Strategy & $\left(V_{A}, \hat{L}\right)_{k=1,2, . . N s}$ & $\hat{\omega}_{i}, i=1,2,3$ \\
\hline Max. $\hat{\omega}_{1}$ & $(0.650,0.3375)_{1},(0.1885,0.1625)_{2}$ & $(3.454,5.537,8.081)$ \\
\hline Max. $\hat{\omega}_{2}$ & $(0.405,0.3650)_{1},(0.7570,0.135)_{2}$ & $(2.820,6.473,8.914)$ \\
\hline Max. $\sum_{i=1}^{3} \alpha_{i} \hat{\omega}_{i}$ & $(0.750,0.1450)_{1},(0.3975,0.355)_{2}$ & $(3.289,6.471,9.468)$ \\
\hline
\end{tabular}

Table (6): Optimal patterns with linear and parabolic distributions of $V_{A}(\hat{x})$. Material distribution is symmetrical about the middle of bar length.

\begin{tabular}{|l|l|l|l|}
\hline Type & Strategy & Optimal $\mathrm{V}_{\mathrm{A}}(\hat{x})$ & $\hat{\omega}_{i}, i=1,2,3$ \\
\hline \multirow{3}{*}{ Linear } & Max. $\left(\hat{\omega}_{1}\right)$ & $1.0-2 \hat{x}$ & $\{3.5922,4.9481,8.3353\}$ \\
\cline { 2 - 4 } & Max. $\sum_{i=1}^{3} \alpha_{i} \hat{\omega}_{i}$ & $0.75-\hat{x}$ & $\{3.4416,6.0931,9.3144\}$ \\
\hline \multirow{2}{*}{ Parabolic } & Max. $\left(\hat{\omega}_{1}\right)$ & $1.0+3\left(\hat{x}^{2}-\hat{x}\right)$ & $\{3.4706,6.1781,9.0588\}$ \\
\cline { 2 - 4 } & Max. $\sum_{i=1}^{3} \alpha_{i} \hat{\omega}_{i}$ & $0.75+1.5\left(\hat{x}^{2}-\hat{x}\right)$ & $\{3.3566,6.3231,9.3781\}$ \\
\hline
\end{tabular}

\section{Conclusions:}

In view of the importance of enhancing the dynamic performance and raising the overall (stiffness/mass) level of a FGM bar in axial motion, an appropriate optimization model has been formulated for both continuous and discrete distributions of the volume fractions of the selected composite material. The objective function has been measured by maximizing a weighted-sum of the system frequencies while maintaining the total structural mass constant. The weighting factors are best determined from the fact that each frequency ought to be 
maximized from its initial reference value of the uniform baseline design. Optimization of multi-segment Fixed-Fixed bars has indicated that good patterns should be symmetrical about the mid span of the bar. The given exact structural analysis leads to the exact frequencies no matter the number of segments is. It has been confirmed that the segment length is most significant design variable in the whole optimization process. Some investigators who apply finite elements have not recognized that the length of each element can be taken as a main design variable in the whole set of optimization variables. It has also been shown that normalization of all terms results in a naturally scaled objective function, constraints and design variables, which is recommended when applying different optimization techniques. The results from the present approach reveals that piecewise grading of the material can be promising producing truly efficient bar designs with enhanced dynamic performance. In conclusion, a powerful design tool has been obtained by formulating an appropriate objective function and applying mathematical programming techniques to the resulting optimization problem. Other secondary effects such as material and geometrical nonlinearities due to large deformation shall be investigated in future studies.

\section{References:}

[1] S. Suresh and A. Mortensen, Fundamentals of functionally graded materials. Cambridge University Press, 1998.

[2] C.T. Loy, K.Y. Lam, J.N. Reddy, Vibration of functionally graded cylindrical shells, Int. J. Mech. Sci., Vol. 3, P. 309-324, 1999.

[3] I. Elishakoff and Z. Guede, Analytical polynomial solutions for vibrating axially graded beams, J. Mech. Adv. Mater. Struct. , Vol.11, P.517-533, 2004.

[4] S.S. Vel and R.C. Batra, Three-dimensional Exact solution for the vibration of functionally graded rectangular plates, Journal of Sound and Vibration, Vol. 272, P.703-730, 2004.

[5] J. A. Masad, Optimization methods with structural dynamics applications, Journal of Computers and Structures, Vol. 62, No.3, P.521-525, 1997.

[6] K.Y. Maalawi, Optimal mass and frequency design of rods in longitudinal vibrations, $6^{\text {th }}$ Conference on theoretical and applied mechanics, Academy of Scientific Research and Technology, Cairo, Egypt, P.259-273, March 3-4, 1999.

[7] L.F. Qian and R.C. Batra, Design of bidirectional functionally graded plate for optimal natural frequencies, Journal of Sound and Vibration, Vol.280, P.415-424, 2005.

[8] A.J. Goupee and S.S.Vel, Optimization of natural frequencies of bidirectional functionally graded beams, Journal of Structural and Multidisciplinary Optimization, Vol.32, No.6, P.473484, 2006.

[9] L. Librescu and K. Maalawi, Material grading for improved aeroelastic stability in composite wings, Journal of Mechanics of Materials and Structures, Vol.2, No.7, P.1381-1394, 2007.

[10] L. Meirovitch, Principles and Techniques of Vibrations, Englewood Cliffs: NJ, Prentice-Hall, 1997.

[11] I.M. Daniel and O. Ishai, Engineering mechanics of composite materials, $2^{\text {nd }}$ ed., Oxford Univ. Press, New York, 2006.

[12] C.H. Edwards and D.E. Penney, Differential equations and boundary value problems: computing and modeling, Prentice Hall, Englewood Cliffs, NJ, 2004.

[13] K.Y. Maalawi and N.M. El-Chazly, Global optimization of multi-element beam-type structures, The $2^{\text {nd }}$ International Conference on Advances in Structural Engineering and 
Proceeding of the $14^{\text {th }}$ AMME Conference, $25-27$ May $2010 \quad$ Paper DV - $2 \quad 11$

Mechanics, ASEM02, Busan, South Korea, 2002.

[14] P. Venkataraman, Applied Optimization with MATLAB Programming, John Wiley \& Sons, Inc., 2002. 